\title{
Biomechanical properties of heel-pad and metatarsal head soft tissue and foot ulcers in patients with systemic sclerosis: a case control study
}

\author{
Mandana Pourian $^{1 *}$, Iman Mohseni ${ }^{1}$, Maryam Biglari Abhari ${ }^{2}$, Mahbod Sianpanah ${ }^{3}$
}

${ }^{1}$ Radiology Department, Firouzgar Hospital, Iran University of Medical Sciences, Tehran, Iran. ${ }^{2}$ Firouzgar Hospital, Iran University of Medical Sciences, Tehran, Iran. ${ }^{3}$ Community Medicine Department, Internal Medicine Department, Shariati hospital, Tehran University of Medical Sciences, Tehran, Iran.

\begin{abstract}
Systemic sclerosis is a chronic connective tissue disease accompanied with skin and inner organ fibrosis and increased risk of foot ulcers. Biomechanical indexes (soft tissue thickness and compressibility) could affect the risk of this phenomenon.

This study aimed to assess the heel pad and first metatarsal head soft-tissue thickness and compressibility index in scleroderma patients with and without foot ulcers and compare them with those of healthy individuals.

Heel pad thickness in standing (loaded) and lying (unloaded) positions was measured in 40 scleroderma patients (diagnosed based on American College of Rheumatology classification criteria) by means of lateral foot radiography. The compressibility index was measured as the ratio of loaded to unloaded thickness. Also, soft-tissue thickness of the first metatarsal head was measured with ultrasound. Results were compared with 40 healthy controls of matched age and body mass index.

Among 40 scleroderma patients ( 36 females, 4 males) with a mean age of $45( \pm 12)$ years, a mean body mass index of $25.5( \pm 4)$, and a mean disease duration of 10 years $( \pm 9.6), 8(20 \%)$ patients had foot ulcers. Heel pad thickness $(P$ value $=0.03)$ and the compressibility index $(P$ value $=0.005)$ in the dominant side and the metatarsal head soft-tissue thickness on both sides $(P$ value $<0.0001)$ had significant differences with the control group. When comparing patients with and without foot ulcers, the soft-tissue thickness in both heels was significantly different ( $P$ value 0.01 for the right side and 0.007 for the left side). Thicknesses were negatively related to, but no relationship between the compressibility index and disease duration was observed.

Plantar soft-tissue thickness decreases in scleroderma patients and could be a risk factor for foot ulcers.
\end{abstract}

Keywords: systemic sclerosis, ulcer, heel pad, biomechanics

\section{Introduction}

Systemic sclerosis $(\mathrm{SSc})$ is a connective tissue disorder characterized by three principal features: vasculopathy, immune activation with the production of autoantibodies and alterations in immune cells, and fibroblast dysfunction with excessive matrix deposition leading to fibrosis of the skin and internal organs [1,2].

The skin is one of the main organs affected by systemic sclerosis. Studies have shown that the extent, distribution, and rate of progression of skin involvement in SSc correlate with internal organ involvement and patient outcome $[3,4]$. Skin disease is both a disabling feature of SSc [5] and a predictor of visceral involvement and increased mortality [6, 7].

A number of methods for evaluating skin involvement in systemic sclerosis have been introduced. The modified Rodnan skin score (mRSS) is used in most clinical centers; however, this method is qualitative, and its validity and reproducibility could be distorted by intra- and interobserver variability (18\% and $21 \%$, respectively) [8]. Hence, quantitative methods are preferred for the evaluation of skin thickness and stiffness.

The impact of SSc on foot health has been poorly studied. The few published studies have shown that podiatric problems are common and potentially disabling for patients with SSc. The biomechanical properties of the foot also seem to be altered in SSc [9].

The primary goal of the current study was to evaluate changes in soft-tissue thickness and stiffness (using a compressibility index) of both the heel-pad and the first metatar-

Personal non-commercial use only.Rheumatology Research Journal. Copyright (C) 2019. All rights reserved

*Corresponding Author: Mandana Pourian, MD, Radiologist, Firouzgar Hospital, Iran University of Medical Sciences, Radiology Department, Tehran, Iran. Email: mandanapourian@gmail.com, Tel: (+98) 9391450830

Received: 30 November 2018 ; Accepted: 16 February 2019 
sal head (which are more affected by biomechanical alterations in the foot) in SSc patients through the inexpensive and widely available imaging methods of ultrasound and X-ray, to better predict foot ulcers in the course of the disease.

\section{Materials and Methods}

\section{Study design}

This study was a case-control study of patients attending the rheumatology clinic at Firouzgar Hospital, Iran University of Medical Sciences, from October 2016 to March 2017. Before starting the study, ethical approval was obtained from the Ethics Committee of Iran University of Medical Sciences.

\section{Study population}

Forty patients with systemic sclerosis who fulfilled the inclusion criteria below were enrolled in this study:

1. Diagnosis of systemic sclerosis made by a rheumatologist based on diagnostic criteria of The American College of Rheumatology (ACR);

2. Aged between 20 and 70 years;

3. No history of diabetes mellitus.

Patients with uncertain diagnoses, aged out of range, or problems possibly affecting the biomechanical properties of the foot, e.g., a history of diabetes mellitus or vasculitis, foot deformity, or smoking (because of vascular side ef- fects), were excluded.

Forty healthy age- and BMI-matched volunteers who fulfilled the inclusion criteria were also enrolled in the study for comparison purposes.

All participants gave informed consent prior to the study. Clinical data and disease history were abstracted from patients' medical records in the rheumatology clinic.

\section{Image acquisition}

Lateral foot radiographs of both feet (with a focal-film distance of 40 inches using $45 \mathrm{KV}$ and 5 MAS [125 mA, $0.04 \mathrm{sec}]$ ) were utilized for the evaluation of both unloaded (lying) and loaded (standing) soft-tissue thickness of the heel-pad in both patients and healthy subjects. Loaded radiographs were obtained after 8 seconds of weight-bearing on the target foot in a standing position. The soft-tissue density under the surface of the calcaneus was measured to determine heel-pad thickness. The measurement was made from the lowest part of the plantar tuberosity of the calcaneus vertically to the skin edge (Figure 1).

The compressibility index (CI) was defined as the ratio of the heel-pad thickness in loaded position to heel-pad thickness in unloaded positions; as the index approaches one, the elasticity approaches zero. All measurements were made by one radiologist. The radiographic images of a sample patient are shown in Figure 2.

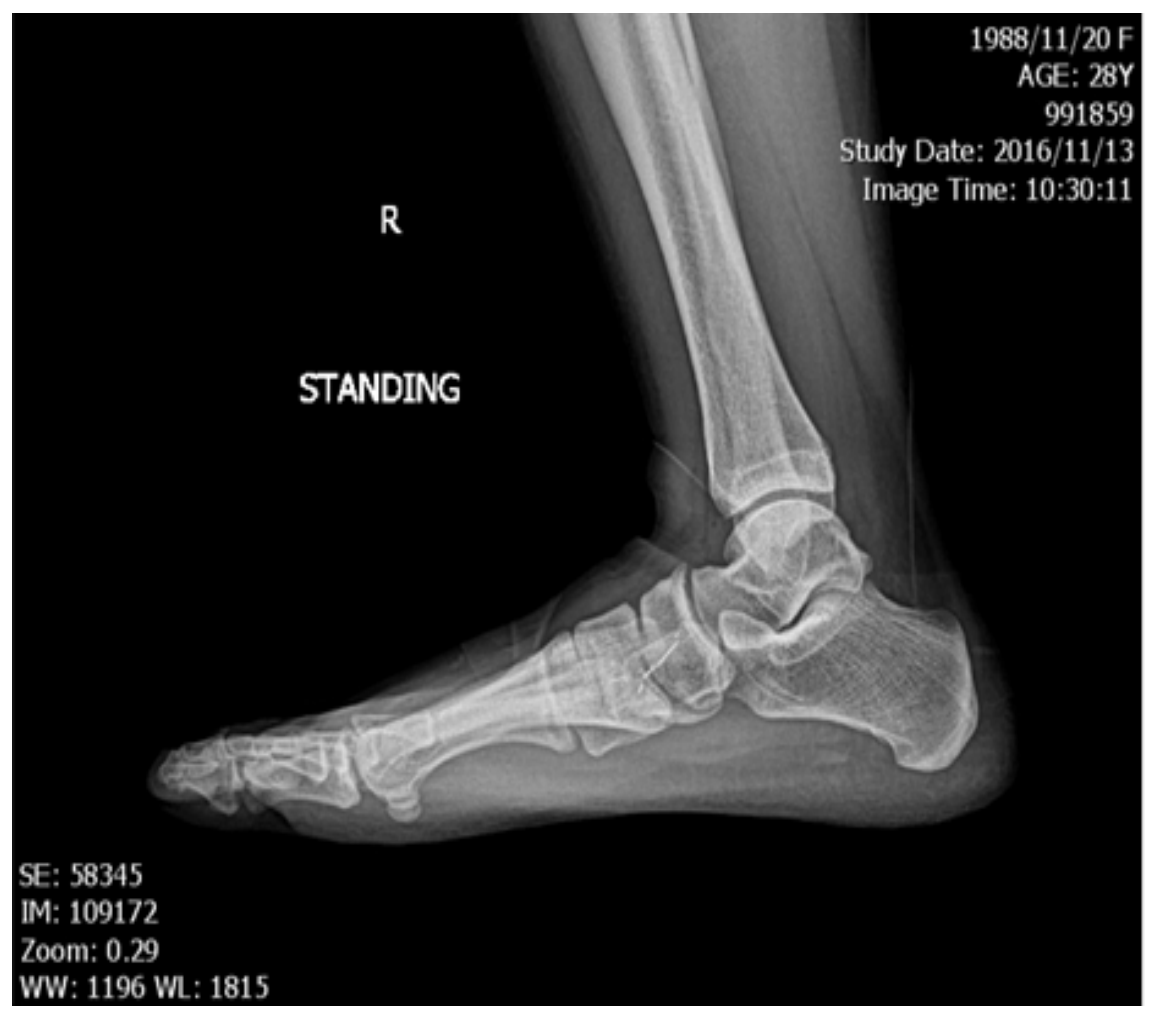

Figure 1. Sample measurement of soft-tissue thickness of heel pad using lateral foot X-ray 

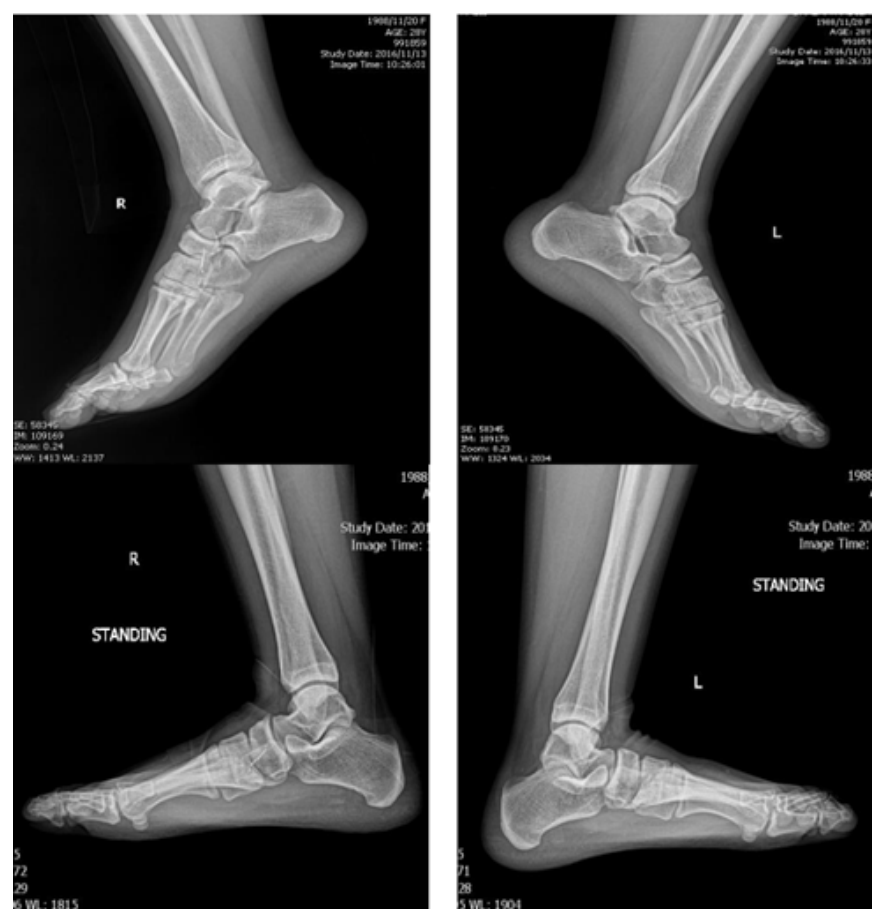

Figure 2. Lateral foot X-ray of both feet in lying (unloaded) and standing (loaded) positions

A sonographic study of the first metatarsal head was also done to measure the soft-tissue thickness. Ultrasound was used for this part of the study because of limitations of the radiography due to the overlapping of the soft tissue of the metatarsal heads on the lateral foot X-ray. Each subject was placed in a sitting position with the ankle in the neutral position and the knee in the extended position lying on the examiner's thigh. All measurements were obtained by
Mindray DC-7 ultrasound machine with $7 \mathrm{MHz}$ transducer. An adequate amount of ultrasound gel was applied to prevent transducer pressure impairment of the evaluation. Soft-tissue thickness, which was the shortest distance between the first metatarsal head and the skin surface, was measured. All measurements were made by the same operator (Figure 3).

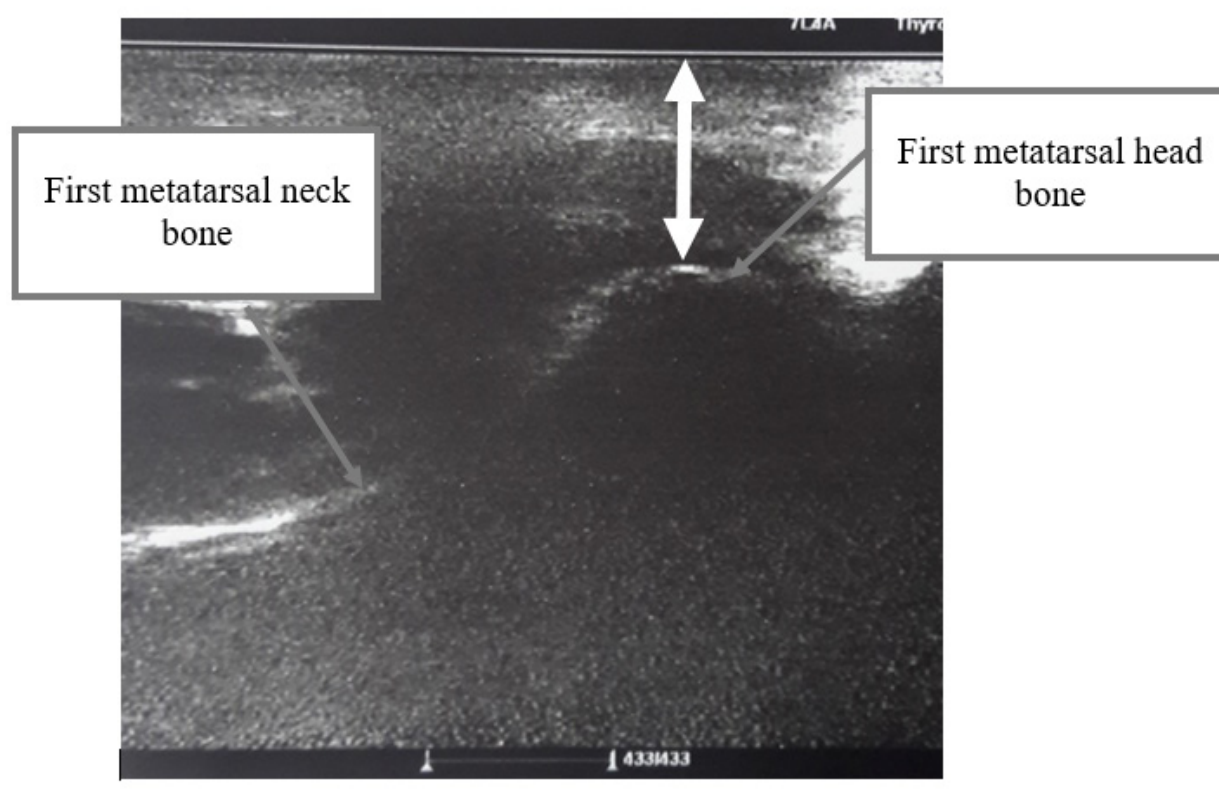

Figure 3. Measurement of first metatarsal head soft-tissue thickness with ultrasound 


\section{Statistical Analysis}

Statistical analysis was performed using IBM SPSS Statistics, version 18. The chi-square test was used for the comparison of nominal and categorical variables, and the independent sample T-test was used for quantitative measurements.

The soft-tissue thickness of the heel pad of both feet (dominant and non-dominant) was measured in lateral foot radiographs. The compressibility index was calculated using the formula, and the soft-tissue thickness of the first metatarsal head of both feet was obtained using the ultrasound technique. These variables as well as demographic data (age,
BMI, gender, disease duration, age of disease onset) were compared between the control and patient groups, and also between patients with and without foot ulcers.

\section{Results}

The average age and BMI distribution between the two groups was similar. The demographic data is summarized in Table 1. The average age of patients at disease onset was $36 \pm 11$ (23-67) years, the mean duration of disease was 10 years. Among the patients, $57 \%$ had objective Raynaud's phenomenon, and $20 \%$ had foot ulcer (Table 2). Except for one patient, the rest were right-handed.

Table 1. Demographic characteristics of normal subjects and patients

\begin{tabular}{lccc}
\hline & Patients & Controls & P value \\
\hline No. of subjects & 40 & 40 \\
Gender & 4 male $(10 \%)$ & 10 male (25\%) \\
Age (years), Mean (SD) & 36 female & 30 female & 0.25 \\
BMI, Mean (SD) & $45(12.3)$ & $42(11.5)$ & 0.45 \\
Disease duration (years) & $25.5(3.8)$ & $26.1(3.7)$ & \\
Age at disease onset (years) & $10 \pm 9.6$ & & \\
\hline
\end{tabular}

Table 2. Disease characteristics of patients

\begin{tabular}{cc}
\hline Characteristic & No. (\%) \\
\hline Foot ulcer & $8(20 \%)$ \\
Objective Raynaud phenomenon & $23(57 \%)$ \\
Subjective Raynaud phenomenon & $24(60 \%)$ \\
\hline
\end{tabular}

\section{Thickness and compressibility index measurement}

In the current study, heel-pad thickness (17.8 vs $19 \mathrm{~mm})$ and first metatarsal soft tissue thickness (10.5 vs $12 \mathrm{~mm})$ were significantly lower in the dominant foot of patients in comparison with normal subjects. The compressibility index of the heel pad was significantly higher in SSc patients than in normal individuals ( $\mathrm{p}$-value $<0.05$ ) (Table 3 ).

The same significant difference was seen in the heel-pad thickness of both feet (15.5 vs $18.4 \mathrm{~mm}$ in the right foot and 15 vs 18.7 in the left foot) among SSc patients with or without ulcer, but neither the metatarsal head thickness nor the compressibility index differed in either group (Table 4).
The heel-pad thickness was overall $6 \%$ less in scleroderma patients than normal subjects (17.8 vs. $19 \mathrm{~mm})$; the difference was $18 \%$ in patients with a foot ulcer (15.5 vs. 19 $\mathrm{mm}$ ) and $3 \%$ in patients without foot ulcers. The overall difference in the compressibility index was $9 \%(0.64$ in healthy subjects vs. 0.7 in scleroderma patients), with the same distribution among patients with and without foot ulcers.

Among demographic factors evaluated in this study, BMI and disease duration demonstrated a positive correlation with heel-pad thickness, but none of them were significantly correlated with the compressibility index. Gender and age demonstrated no significant correlation with any measurements $(25 \%$ of male patients and $19 \%$ of female patients had ulcers). 
Table 3. Comparison of heel-pad thickness, compressibility index, and metatarsal head soft-tissue thickness between normal subjects and SSc patients (mean, standard deviation)

\begin{tabular}{lccc}
\hline & $\begin{array}{c}\text { Scleroderma } \\
\text { Mean (SD) }\end{array}$ & $\begin{array}{c}\text { Control } \\
\text { Mean (SD) }\end{array}$ & P value \\
\hline Right heel pad unloaded thickness radiograph (mm) & $17.8(3)$ & $19(1.9)$ & 0.03 \\
Right heel pad compressibility index & $0.7(0.07)$ & $0.64(0.08)$ & 0.005 \\
Left heel pad unloaded thickness radiograph (mm) & $17.96(3.4)$ & $0.65(0.08)$ & 0.1 \\
Left heel pad compressibility index & $0.68(0.08)$ & $12.5(1.6)$ & 19.98 \\
Right metatarsal head soft-tissue thickness US (mm) & $10.7(2)$ & $10(1.9)$ & $<0.0001$ \\
Left metatarsal head soft-tissue thickness US (mm) & $1.5)$ & $<0.0001$ \\
\hline
\end{tabular}

Table 4. Comparison of heel-pad thickness, compressibility index, and metatarsal head soft-tissue thickness between patients with and without foot ulcers (mean, standard deviation)

\begin{tabular}{lccc}
\hline \multicolumn{1}{c}{.. } & $\begin{array}{c}\text { With ulcer } \\
\text { Mean (SD) }\end{array}$ & $\begin{array}{c}\text { Without ulcer } \\
\text { Mean (SD) }\end{array}$ & P value \\
\hline Right heel pad unloaded thickness radiograph (mm) & $15.5(3)$ & $18.4(2.7)$ & 0.01 \\
Right heel pad compressibility index & $0.71(0.09)$ & $0.7(0.07)$ & 0.72 \\
Left heel pad unloaded thickness radiograph (mm) & $15(2.8)$ & $18.7(3.2)$ & 0.007 \\
Left heel compressibility index & $0.71(0.08)$ & $0.67(0.08)$ & 0.18 \\
Right metatarsal head soft-tissue thickness US (mm) & $10.8(2.4)$ & $10.7(2)$ & 0.86 \\
Left metatarsal head soft-tissue thickness US (mm) & $9.3(1.4)$ & $10.2(1.7)$ & 0.27 \\
\hline
\end{tabular}

\section{Discussion}

As foot ulcers are one of the irritating complications of scleroderma that have a high impact on the functional disability of patients, early detection and risk factor modification are necessary. The foot is a specific organ, because mechanical (external) forces affect soft-tissue changes.

A significant change was found in the compressibility index and soft-tissue thickness of the feet in scleroderma patients. With disease progression over time, the thickness decreases, but the compressibility index does not seem to change significantly. To justify these findings, it was hypothesized that as the scleroderma progresses and becomes more severe over time, soft tissue becomes stiffer pathophysiologically because of more extra-cellular matrix accumulation and because the variation range becomes narrower. Finally, changes in the stiffness could hardly be detected with the method of measurement used in this study.

In the current study, heel-pad thickness and first metatarsal soft-tissue thickness were significantly lower in the dominant foot of patients in comparison with normal subjects, but the relationship was not significant in the non-dominant foot. This could be due to the higher pressure on the dominant foot.

In addition to the main findings, two ancillary points were detected. The first point was that Raynaud's phenomenon had a significant relationship with ulcer manifestation $(P$ value $=0.006)$ (as Sunderkötter et al. also found) [12], but did not have a significant correlation with quantitative parameters (thickness and compressibility index). This is reasonable because of the common vasculopathic nature of foot ulcers and Raynaud's phenomenon. The second point was identified when the group of patients without ulcers was compared with the normal control group; the compressibility index increased $(0.7$ vs 0.65$)$, but thicknesses had no significant difference (there was a 0.3-0.6 millimeters increase in thickness, which is not significant statistically). This could mean that the change in stiffness occurs prior to the change in thickness. Thus, stiffness is a more important factor than skin thickness for the early detection of skin changes in patients with SSc.

The results can be applied for the patients' clinical follow-up, so the compressibility changes can be more 
valuable in their first clinician visits. However, with the disease progressing in severity and time, following the patients with thickness measurements (mainly heel-pad) can be more prognostic and a better predictor of foot ulcers. Unfortunately, no cut-off point for thicknesses could be found for which less than that, the risk of foot ulcers would significantly increase. It is much more valuable to compare measurements between visits.

A thorough search of the literature revealed no study similar to this one on the biomechanics of the foot in scleroderma patients, though similar studies on normal populations and specific groups with foot problems (e.g., sportsmen with podiatric problems, patients with heel pain, diabetic patients with foot ulcers, etc.) are available. Several studies have addressed digital ulcers in scleroderma patients and their possible risk factors. The study of Allanore et al. is one of the largest and most recent (2016) studies done on digital ulcers and gangrene with 4642 patients with systemic sclerosis. They concluded that being a current/former smoker and having a previous limb sympathectomy were independent risk factors for gangrene of the fingers [10]. Smokers were excluded from the current study, because of the possible effects smoking could have on vessels interfering with the study elements.

A number of studies suggested male gender [11, 12] and younger age at onset [11-14] as risk factors for digital ulcers. No significant relationship between gender and digital ulcers was found in the current study, possibly due to the small number of male subjects. Duration of disease had a positive relationship with ulcers in the current study, in agreement with Thiev [11], but younger age of onset was not found to be a related factor in the current study.

Several measurement methods for determining skin properties in scleroderma have been introduced. Moinzadeh et al. (2012) collected different measurement types for skin involvement [15]. The most widely-used procedure is the modified Rodnan skin score (mRSS), which qualitatively grades skin elasticity through palpation of 17 parts of the body by a clinician (not including the sole of the foot). Because of operator dependency, this test is prone to inter-/intra-observer variability. Moreover, it is not sensitive to initial and mild skin changes.

Two other tools in this area are the plicometer and durometer tests, which are more reproducible and objective techniques. The plicometer test works similarly to clinical pinching of the skin in mRSS measurements. The durometer test, on the other hand, measures the hardness (but not the thickness) of the skin through applied pressure $[16,17]$.

Magnetic resonance imaging (MRI) is a modality that has been used mostly to study musculoskeletal involvement, e.g., arthritis and myositis. The weak point of this method is its cost, which precludes its extensive use.

Ultrasound is a cost-effective, easy-to-use, quantitative technique which can measure not only skin thickness, but also other properties of the skin, like the characteristics of subcutaneous connective tissue which may occur prior to changes in the skin's thickness [18].

A few studies used the ultrasound technique to measure skin thickness and other properties in scleroderma patients. Moore et al. studied the sites which are normally evaluated for mRSS [19]. Kaloudi et al. (2010) used this technique to measure skin thickness in hand digits, which are prone to ulcers as well [20]. Suliman et al. (2018) recently characterized skin ulcers by means of the ultrasound technique (grayscale and Doppler study) [21]. Studies have shown reliable results with good inter-/intra-observer variability of this imaging method [22].

While the current study investigated a number of biomechanical indices in patients with scleroderma and made comparisons with age- and BMI-matched healthy subjects, it has some limitations. Theoretically, foot ulcers are affected by biomechanical (e.g., weight pressure, type of footwear) and ischemic factors. The effects of each of these factors could not be calculated separately. Patients unable to stand on their feet were not enrolled in the study because of either painful foot ulcers or general not well-being. In effect, patients with more severe disease were not investigated. Another weak point of this study was that patients with and without digital ulcers were not matched based on their medical treatments. Therefore, possible effects of drugs on the biomechanical properties of foot were not considered. Finally, because of the cross-sectional nature of the current study, the course of change in thicknesses and compressibility indices over time could not be seen. This can be a more valuable index than a static measurement in predicting digital ulcers and disease progression. A prospective study will be of great interest to follow up patients through the course of their disease to find the cause and effect relationship of relevant factors.

\section{Conclusion}

Digital ulcers are disturbing complications of scleroderma and have a high impact on the functional disability of patients as well as a direct relationship with internal organ involvement. The current study tried to look at the mechanical side of this catastrophic problem. Because of the high morbidity and great burden, finding precursors and risk factors before the manifestation of an ulcer draws more attention.

Ultrasound and conventional radiography are two inexpensive imaging modalities used to evaluate soft-tissue thickness and the compressibility index, which change in patients with systemic sclerosis, and could be predictors 
of digital ulcers and the overall morbidity and mortality of the disease. Future studies can further investigate the cause and effect of foot ulcers and the biomechanical alterations of the soft tissue. The effects of therapeutic drugs on these quantitative changes could also be a possible area for future investigation.

\section{Acknowledgments}

The authors thank all those who helped them writing this paper.

\section{Conflict of Interest}

The authors declare no conflicts of interest. 


\section{References}

1. Eckes B, Wang F, Moinzadeh P, Hunzelmann N, Krieg T. Pathophysiological Mechanisms in Sclerosing Skin Diseases. Front Med (Lausanne) 2017; 4:120. doi: 10.3389/ fmed.2017.00120.

2. Nihtyanova SI, Brough GM, Black CM, Denton CP. Clinical burden of digital vasculopathy in limited and diffuse cutaneous systemic sclerosis. Ann Rheum Dis 2008; 67(1):120-23. doi: 10.1136/ard.2007.072686.

3. Clements PJ, Hurwitz EL, Wong WK, Seibold JR, Mayes $\mathrm{M}$, et al. Skin thickness score as a predictor and correlate of outcome in systemic sclerosis: high-dose versus low-dose penicillamine trial. Arthritis Rheum 2000; 43(11):244554. doi: 10.1002/1529-0131(200011)43:11<2445::aid-anr11>3.0.co;2-q.

4. Clements PJ, Lachenbruch PA, Ng SC, Simmons M, Sterz M, Furst DE. Skin score. A semi-quantitative measure of cutaneous involvement that improves prediction of prognosis in systemic sclerosis. Arthritis Rheum 2010; 33(8):1256-63. doi: 10.1002/art.1780330828.

5. Guillevin L, Hunsche E, Denton CP, Krieg T, Schwierin B, Rosenberg D,et al. Functional impairment of systemic scleroderma patients with digital ulcerations: results from the DUO Registry. Clin Exp Rheumatol 2013; 31(2 Suppl 76):71-80.

6. Bruni C, Guiducci S, Bellando-Randone S, Lepri G, Braschi F, Fiori G, et al. Digital ulcers as a sentinel sign for early internal organ involvement in very early systemic sclerosis. Rheumatology (Oxford) 2015; 54(1):72-76. doi: 10.1093/rheumatology/keu296.

7. Mihai C, Landewé R, van der Heijde D, Walker UA, Constantin PI, Gherghe AM, et al. Digital ulcers predict a worse disease course in patients with systemic sclerosis. Ann Rheum Dis 2016; 75(4):681-86. doi: 10.1136/annrheumdis-2014-205897.

8. Clements P1, Lachenbruch P, Siebold J, White B, Weiner $\mathrm{S}$, Martin R, et al. Inter and intraobserver variability of total skin thickness score (modified Rodnan TSS) in systemic sclerosis. J Rheumatol 1995; 22(7):1281-85.

9. Bongi SM, Ravenni G, Ciampi B, Del Rosso A, El Aoufy $\mathrm{K}$. Biomechanical podiatric evaluation in an Italian cohort of patients with systemic sclerosis: A pilot study. Eur J Rheumatol 2016; 3(4):169-74. doi: 10.5152/eurjrheum.2016.053.

10. Allanore Y, Denton CP, Krieg T, Cornelisse P, Rosenberg D, Schwierin B, et al. Clinical characteristics and predictors of gangrene in patients with systemic sclerosis and digital ulcers in the Digital Ulcer Outcome Registry: a prospective, observational cohort. Ann Rheum Dis 2016; 75(9):1736-40. doi: 10.1136/annrheumdis-2016-209481.

11. Tiev KP, Diot E, Clerson P, Dupuis-Siméon F, Hachulla E, Hatron PY, et al. Clinical features of scleroderma patients with or without prior or current ischemic digital ulcers: post-hoc analysis of a nationwide multicenter cohort (ItinérAIR-Sclérodermie). J Rheumatol 2009; 36(7):1470-76. doi: 10.3899/jrheum.081044.
12. Sunderkötter C, Herrgott I, Brückner C, Moinzadeh P, Pfeiffer C, Gerss J, et al. Comparison of patients with and without digital ulcers in systemic sclerosis: detection of possible risk factors. Br J Dermatol 2009; 160(4):835-43. doi: 10.1111/j.1365-2133.2008.09004.x.

13. Khimdas S, Harding S, Bonner A, Zummer B, Baron M, Pope J. Associations with digital ulcers in a large cohort of systemic sclerosis: results from the Canadian Scleroderma Research Group registry. Arthritis Care Res (Hoboken) 2011; 63(1):142-49. doi: 10.1002/acr.20336.

14. Hachulla E, Clerson P, Launay D, Lambert M, Morell-Dubois $\mathrm{S}$, Queyrel V, et al. Natural history of ischemic digital ulcers in systemic sclerosis: single-center retrospective longitudinal study. J Rheumatol 2007; 34(12):242330.

15. Moinzadeh P, Denton CP, Abraham D, Ong V, Hunzelmann N, Eckes B, et al. Biomarkers for skin involvement and fibrotic activity in scleroderma. $\boldsymbol{J}$ Eur Acad Dermatol Venereol 2012; 26(3):267-76. doi: 10.1111/j.14683083.2011.04206.x.

16. Chao CY, Zheng YP, Cheing GL. Epidermal thickness and biomechanical properties of plantar tissues in diabetic foot. Ultrasound Med Biol 2011; 37(7):1029-38. doi: 10.1016/j.ultrasmedbio.2011.04.004.

17. Czirják L, Foeldvari I, Müller-Ladner U. Skin involvement in systemic sclerosis. Rheumatology (Oxford) 2008; 47 Suppl 5: v44-5. doi: 10.1093/rheumatology/ken309.

18. Hesselstrand R, Scheja A, Wildt M, Akesson A. High-frequency ultrasound of skin involvement in systemic sclerosis reflects oedema, extension and severity in early disease. Rheumatology (Oxford) 2008; 47(1):84-87. doi: 10.1093/rheumatology/kem307.

19. Moore TL, Lunt M, McManus B, Anderson ME, Herrick AL. Seventeen-point dermal ultrasound scoring system--a reliable measure of skin thickness in patients with systemic sclerosis. Rheumatology (Oxford) 2003; 42(12):155963. doi:10.1093/rheumatology/keg435.

20. Kaloudi O, Bandinelli F, Filippucci E, Conforti ML, Miniati I, Guiducci S, et al. High frequency ultrasound measurement of digital dermal thickness in systemic sclerosis. Ann Rheum Dis 2010; 69(6):1140-43. doi: 10.1136/ ard.2009.114843.

21. Suliman YA, Kafaja S, Fitzgerald J, Wortsman X, Grotts $\mathrm{J}$, Matucci-Cerrinic M, et al. Ultrasound characterization of cutaneous ulcers in systemic sclerosis. Clin Rheumatol 2018; 37(6):1555-61. doi: 10.1007/s10067-018-3986-5.

22. Kang T, Abignano G, Lettieri G, Wakefield RJ, Emery P, Del Galdo F. Skin imaging in systemic sclerosis. Eur $\boldsymbol{J}$ Rheumatol 2014; 1(3):111-16. doi: 10.5152/eurjrheumatol.2014.036. 